\title{
Report on Unusual Sites of Lymph Node Metastases in Nasopharyngeal Carcinoma
}

\author{
Dang Nguyen Van ${ }^{a}$ b Thao Bich Nguyen ${ }^{c} \quad$ Nhung Thu Nguyen Thi ${ }^{b}$ \\ Quang Le Van ${ }^{a}$ \\ a'Department of Oncology, Hanoi Medical University, Hanoi, Vietnam; ${ }^{b}$ Department of \\ Head and Neck Radiation Oncology, Vietnam National Cancer Hospital, Hanoi, Vietnam; \\ cOncology Center, Phu Tho General Hospital, Phu Tho Province, Viet Tri, Vietnam
}

\author{
Keywords \\ Nasopharyngeal cancer · Lymph node metastasis · Parotid gland metastasis · Cheek \\ metastasis
}

\begin{abstract}
Nasopharyngeal carcinoma (NPC) is amongst the most common malignancies of head and neck cancers. Most patients are admitted to the hospital with advanced disease. NPC has a tendency toward early metastatic spread to cervical lymph nodes, and levels II and III are most commonly involved. A few reports have indicated specific metastatic sites of nasopharyngeal cancer, including lymph node metastasis and distant metastasis. Evidence of histopathology and immunohistochemistry is required to prove NPC origin. In many cases, surgery can be performed to obtain accurate evidence of the pathology. However, surgery can also affect the overall treatment plan and strategy for NPC and should be considered in the specific circumstances of the disease. Multidisciplinary consultation is required for these uncommonly specific metastases. Paying attention to the specific lymph node metastasis sites of NPC plays an important role in accurately diagnosing the stage, thereby giving an appropriate treatment strategy. It is also important in determining radiotherapy volumes because radiotherapy is the standard therapy for this disease. Herein, we are reporting 2 cases of NPC with clinical metastasis to unusual lymph node sites such as the parotid salivary gland and the cheek. Histological analyses from the resected specimens confirmed its nasopharyngeal origin. Lymph node metastases in the parotid gland and the cheek are unusual. In diagnosis and follow-up, it is necessary to evaluate carefully to make an accurate diagnosis and appropriate treatment plans for patients as well as early detect recurrent metastases at uncommon sites of lymph nodes.
\end{abstract}

\section{Karger $\stackrel{2}{\circ}$}




\section{Introduction}

Nasopharyngeal cancer is the predominant tumor type arising from the nasopharynx. It differs from other head and neck squamous cell carcinomas in epidemiology, histology, natural histology, and response to treatment.

In epidemiological areas such as Southern China, the histologic subtype mainly is undifferentiated carcinoma-type 3 according to the WHO or undifferentiated carcinoma of nasopharyngeal type (UCNT) [1]. According to GLOBOCAN 2020, in Vietnam, nasopharyngeal cancer ranked 6th among the cancers recorded [2]. The risk appears to be due to an interaction of some factors: Epstein-Barr virus infection, environmental factors (such as the high intake of preserved foods and smoking), and genetic predisposition [3]. Diagnosis of NPC is mainly based on endoscopic ENT examination combined with biopsy, particularly some cases of diagnosis are determined by metastases of neck lymph nodes or distant metastases while primary tumors are not detected. At the time of diagnosis, 5-10\% of NPC patients have distant metastases, most of which are bone metastases (64-67\%), liver (32-34\%), lung (15-22\%), and distant lymph node metastases (12-15\%) [4]. Lymph node metastases are present at diagnosis in $85-90 \%$ in unilateral and over $50 \%$ in the bilateral neck. According to a metaanalysis of clinical evidence evaluating patterns of regional lymph node metastasis of NPC (Ho et al. [4]), the results were as follows: $85 \%$ of NPC cases presented with lymphadenopathy. The most commonly involved regions include retropharyngeal (69\%) and level II lymph nodes (70\%). The overall probability of levels III, IV, and V nodal involvement is $45 \%$, $11 \%$, and $27 \%$, respectively. Low-risk node groups include the supraclavicular, levels IA/IB and VI nodes, and parotid nodes with involvement rates at 3\%, 0\%, 3\%, 0\%, and 1\%, respectively. Metastases to the parotid gland and cheek lymph nodes are rare [5]. In the literature, lymph node metastasis in the parotid gland in NPC is $0.6-3 \%$ [6]. The drainage of lymph nodes from the pharynx to the parotid gland and cheek lymph nodes is still undiscovered. In clinical practice, we describe an NPC case of parotid gland lymph node metastasis and another case of recurrent NPC with cheek lymph node metastasis after radical treatment.

\section{Case Report/Case Presentation}

\section{Case 1}

A 57-year-old man was hospitalized in March 2019, diagnosed with NPC, stage cT1N2M0 (TNM classification 2010, 7th edition), and histological analyses from biopsy specimens evaluated UCNT. There were enlarged bilateral lymph nodes, and particularly there was a mass at the location of the left parotid salivary gland measuring $1.5 \mathrm{~cm}$ in diameter. It is difficult to distinguish between a tumor of the parotid salivary gland and an enlarged lymph node in the gland. Ultrasound identified a hypoechoic mass in the left parotid salivary gland. Fine-needle aspiration (FNA) resulted in carcinoma metastatic lymph node. 18FDG-PET/CT showed a mass which was located in the left parotid salivary gland, size $1.5 \mathrm{~cm}$ and $\mathrm{SUV}_{\max }=17$ (Fig. 1). Based on clinical and subclinical results, 2 clinical situations have been posed: NPC accompanied by left parotid salivary gland tumor or metastatic lymph node in the parotid salivary gland. These 2 diagnostic situations would lead to different treatments and prognoses. In the case that this was a metastatic lymph node of NPC, concurrent radiochemotherapy would be the optimal and highly consensual option. However, if there was a second cancer, the treatment strategy would have different options: surgery to treat parotid salivary gland cancer first, then concurrent radiochemotherapy to treat nasopharyngeal cancer, or concurrent radiochemotherapy for NPC with consideration of radiotherapy to lymph nodes of the left parotid salivary gland first and then considering the role of surgery.

\section{Karger'}




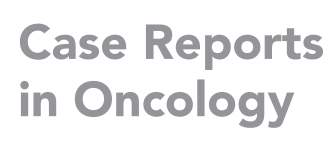

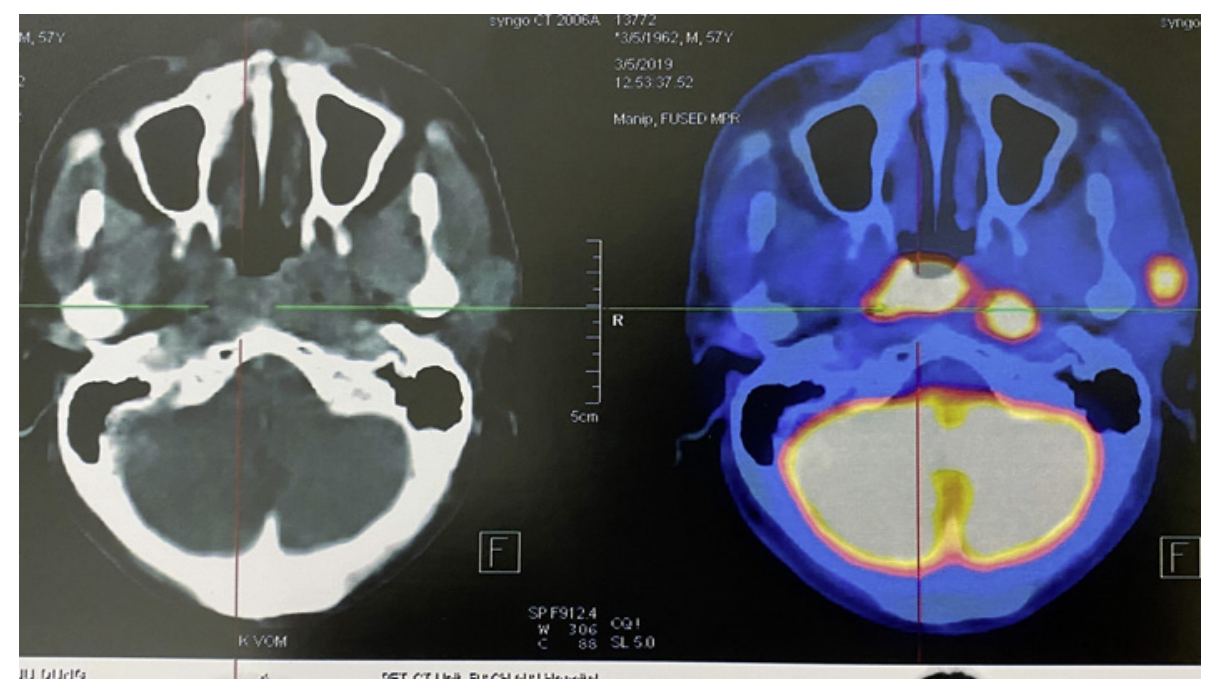

Fig. 1. PET/CT result: increased metabolism in the nasopharynx, paranasal lymph nodes, and left parotid glands.

After multidisciplinary consultation, the parotid mass was removed. The histopathologic analyses showed metastatic lymph nodes with undifferentiated carcinoma, originating from the nasopharynx. Thus, the final diagnosis was NPC with parotid lymph node metastasis. The patient was treated by concurrent radiochemotherapy with a total dose of 69.96 Gy to highrisk volume, 59.4 Gy to medium risk, and 54 Gy to low-risk volume, using the volumetric modulated arc therapy (VMAT) technique and cisplatin $100 \mathrm{mg} / \mathrm{m}^{2}$ every 3 weeks from April 2019 to June 2019. After over 2 years of follow-up, no recurrence or metastasis was found.

\section{Case 2}

A 42-year-old man, in February 2018, was diagnosed with clinical stage cT2N2M0 NPC (TNM classification 2010, 7th edition), and the histopathologic analysis showed UCNT. The patient was treated by concurrent radiochemotherapy with a total dose of 69.96 Gy to highrisk volume, 59.4 Gy to medium risk, and 54 Gy to low-risk volume, using VMAT technique and cisplatin $100 \mathrm{mg} / \mathrm{m}^{2}$ every 3 weeks and 2 cycles of adjuvant cisplatin-5FU from February 2018 to September 2018. After treatment, the patient was re-examined periodically every 3 months. In January 2019 , we found a mass in his right cheek, $0.5 \mathrm{~cm}$, movable, firm, and painless. Ultrasound showed a right cheek lymph node $0.5 \mathrm{~cm}$ is still the umbilical node structure. The patient was followed up and re-examined after 3 months. At the next examination, the patient underwent an ultrasound and FNA. FNA results showed no evidence of lymph node metastasis. Due to being too worried about his condition, the patient suggested that he undergo surgery to remove the lymph node for biopsy. The patient underwent lymphadenectomy, and postoperative histopathologic analyses confirmed its nasopharyngeal origin. After that, he was evaluated by PET/CT, and no lesion was found. He was discharged and had follow-ups periodically. Currently, after over 3 years, the patient is stable with no signs of recurrence or metastasis.

\section{Discussion}

Within the head and neck area, in particular, drainage of parotid lymph nodes is very complex, and there are approximately $20-30$ nodes. The superficial parotid glands have 1-3 nodes, or preauricular lymph nodes, draining lymph from the outside of the ear canal and the

\section{Karger'}


skin of the temples to the posterior neck lymph nodes. Deep parotid nodes in the middle of the parotid gland and along the external jugular vein receive lymph nodes from the skin of the temples, forehead, eyelids, outer and middle ears, parotid glands, and nasal mucosa. All of them drain to the posterior cervical lymph node group. So, the parotid gland is a potential metastatic site of head and neck cancer as well as a distant metastatic site from another location. Metastases to the parotid gland may be via blood, lymph, or direct invasion, or by a combination of the above pathways. Malignant tumors of the face mainly metastasize to the parotid gland, accounting for $80 \%$. Distant metastases to the parotid gland are uncommon; these metastases are usually via blood vessels from the lung, breast, or kidney cancers and salivary parenchyma [5]. Although metastases to parotid lymph nodes are uncommon, they should always be considered in the differential diagnosis of a primary parotid tumor.

NPC metastases to the parotid gland are rare. In the study of Wang et al. [6], 20/1,096 $(1.82 \%)$ NPC patients with parotid gland lymph node metastasis were reported. In this study, the authors also present the high-risk factors to metastases to parotid gland lymph nodes: total diameter of group II lymph nodes $\geq 5 \mathrm{~cm}$ and the presence of other rare cervical lymph node groups [6]. In the situation where the patient with nasopharyngeal cancer is accompanied by lesions in the parotid salivary gland, the following diagnoses are made: lymph node metastasis of nasopharyngeal cancer, primary cancer of the parotid salivary gland, or lymph node metastasis of unknown origin. A definitive diagnosis is often difficult. A comprehensive physical examination is required, and a biopsy of the lymph node mass in the parotid salivary gland is necessary for a definitive diagnosis of lymph node metastasis from NPC. In cases where the histopathological result is an undifferentiated carcinoma, the diagnosis seems easier. However, if the biopsy results are squamous cell carcinoma, the definitive diagnosis is difficult because parotid salivary gland cancer also has a rare incidence of squamous cell carcinoma.

In 2013, DAHANCA, EORTC, HKNPCSG, NCICCTG, NCRU, RTOG, and TGOG agreed on the definition of lymph node grouping for head and neck tumors, including the parotid gland lymph nodes as group VIII. It includes 3 groups: parotid fascia, parotid parenchyma, and preauricular lymph nodes remain extra-fascial and extra-glandular. According to anatomy, parotid lymph nodes receive lymph from the skin of the forehead, temples, eyelids, conjunctiva, outer ear, middle ear, the root of the nose, nasopharynx, and Eustachian tube [7].

These organizations also agreed to define the cheek-face lymph nodes group as group IX, which includes the cheek and face lymph nodes. It consists of superficial lymph nodes surrounding the vein on the outer surface of the buccinator muscle. These nodes extend from the caudal limb (head part) to the lower limit of the lower jaw (caudal part) where the Ib level is found, from the anterior limit of the masseter muscle and the deep Bichat's (posterior) layer to the surface of the facial tissue. Check-face lymph nodes receive veins from the nose, eyes, and cheeks. They are a potential metastatic cancer of the face, nose, and maxillary sinus skin (including cheeks) and the buccinator muscle. However, metastatic cheek lymph nodes in head and neck cancer are uncommon [7].

A retrospective literature review reported 41 cases, of which 22 imaging studies had cheek lymph node metastases. Primary tumors include squamous cell carcinoma and adenocarcinoma of the sinus, tongue, lips, oral mucosa, the skin of the anterior ear, salivary glands, and retromolar trigone. A lymphoma is also a major group for facial lymph node disease. Other cases include lymph node metastases from eye cancer and distant metastases as far as bladder cancer. To our knowledge, there are no reports on metastatic cheek lymph nodes from NPC.

In 2000, Chong and Fan [8] synthesized images taken from 1916 NPC patients (confirmed by histopathology) in the last 5 years. As a result, there were only 8 metastatic facial lymph nodes from NPC in 3 patients $(0.2 \%)$. These include 2 lymph nodes in the buccinator muscle, 2 lymph nodes in the cheeks, 2 lymph nodes under the eye socket, and 1 submandibular lymph node.

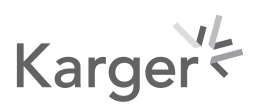


In 2018, Elnaggar et al. [9] found metastatic cheek-face lymph nodes in head and neck cancer in 30 patients ( 22 males and 8 females). The tumor site was the scalp ( $n=3)$, eyelid $(n=2)$, lip $(n=2)$, parotid $(n=6)$, tongue $(n=5)$, alveolar margin $(n=5)$, and buccal mucosa $(n=7)$ and no case from the nasopharynx.

Thus, the drainage metastases from the nasopharynx to the parotid lymph nodes and the cheek lymph nodes are still a question. We explain this by the following 3 mechanisms: (1) via retropharyngeal to parotid parenchyma lymph nodes; (2) indirectly through invasion around the parotid gland; (3) reverse metastases can occur from the metastatic tumors in the neck area, blockage of lymphatic drainage, or via a normal lymphatic drainage system in the neck due to previous invasive treatment intervention.

Because metastases to the cheek lymph nodes are uncommon, they are often overlooked on clinical examination. Even ultrasound and FNA can miss these lesions as in the case of the patient we reported. It is essential for clinicians to have a caring attitude toward cheeks lesions in nasopharyngeal cancer patients. In the diagnosis, we may confuse lymph nodes of the parotid gland and the facial region with metastatic lymph nodes from NPC to the parotid gland and facial lymph nodes. Clinically, this differential diagnosis is important because it will lead to different diagnoses and completely different treatments and prognoses. We should use a variety of diagnostic methods such as FNA, immunohistochemistry analyses, or combined with CT, MRI, and PET/CT evaluation, and even in some cases, surgical removal of lymph nodes to confirm histopathology is necessary.

\section{Conclusion}

In summary, lymph node metastases in the parotid gland and the cheek are unusual. There are no specific statistics evaluating prognosis in patients with NPC with metastatic lymph nodes in the parotid gland and the face. In diagnosis and follow-up, it is necessary to evaluate carefully to make an accurate diagnosis and appropriate treatment plans for patients as well as early detect recurrent metastases at uncommon sites of lymph nodes. Our report as shown above is about 2 cases representing abnormal metastases of nasopharyngeal cancer. Thereby, it is recommended that clinicians should pay attention to special metastatic sites to avoid missing these lesions, leading to misdiagnosis and deficiencies in the treatment strategy for nasopharyngeal cancer.

\section{Statement of Ethics}

Ethical approval was not required for this study in accordance with local/national guidelines. Written informed consent was obtained from the patient for publication of this case report and any accompanying images.

\section{Conflict of Interest Statement}

The authors have no conflicts of interest to declare.

\section{Funding Sources}

There are no funding sources for this manuscript.

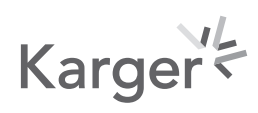




\section{Case Reports in Oncology}

Case Rep Oncol 2021;14:1821-1826

\section{Author Contributions}

Dang Nguyen Van, Radiation Oncologist, treated the patients and wrote this manuscript. Thao Nguyen Bich, Radiation Oncologist, wrote this manuscript. Nhung Nguyen Thi Thu, Radiation Oncologist, revised this manuscript. Quang Le Van, Professor, revised this manuscript.

\section{Data Availability Statement}

The datasets used during the current study are available from the corresponding author on reasonable request.

\section{References}

1 Wu L, Li C, Pan L. Nasopharyngeal carcinoma: a review of current updates. Exp Ther Med. 2018;15(4):368792.

2 Sung H, Ferlay J, Siegel RL, Laversanne M, Soerjomataram I, Jemal A, et al. Global cancer statistics 2020: GLOBOCAN estimates of incidence and mortality worldwide for 36 cancers in 185 countries. Cancer J Clin. 2021;71(3):209-49.

3 Okekpa SI, Mydin MRB, Mangantig E, Azmi NSA, Zahari SNS, Kaur G, et al. Nasopharyngeal carcinoma (NPC) risk factors: a systematic review and meta-analysis of the association with lifestyle, diets, socioeconomic and sociodemographic in Asian region. Asian Pac J Cancer Prev. 2019;20(11):3505-14.

4 Ho FC, Tham IW, Earnest A, Lee KM, Lu JJ. Patterns of regional lymph node metastasis of nasopharyngeal carcinoma: a meta-analysis of clinical evidence. BMC Cancer. 2012;12:98.

5 Zou X, You R, Liu H, He YX, Xie GF, Xie ZH, et al. Establishment and validation of M1 stage subdivisions for de novo metastatic nasopharyngeal carcinoma to better predict prognosis and guide treatment. Eur J Cancer. 2017;77:117-26.

6 Wang H, Cao CN, Luo JW, Yi JL, Huang XD, Zhang SP, et al. High-risk factors of parotid lymph node metastasis in nasopharyngeal carcinoma: a case-control study. Radiat Oncol. 2016;11(11):113.

7 Grégoire V, Ang K, Budach W, Grau C, Hamoir M, Langendijk JA, et al. Delineation of the neck node levels for head and neck tumors: a 2013 update. DAHANCA, EORTC, HKNPCSG, NCIC CTG, NCRI, RTOG, TROG consensus guidelines. Radiother Oncol. 2014;110(1):172-81.

8 Chong VF, Fan YF. Facial lymphadenopathy in nasopharyngeal carcinoma. Clin Radiol. 2000;55(5):363.

9 Elnaggar TM, El-Kased AF, El-Fol HAEK, El-Elaimy MMA, Alzaqri WA, Sabry M. Evaluation of facial lymph node metastasis in head and neck carcinoma. J Clin Case Rep Trials. 2018;1(1):13-7.

\section{Karger'}

